\title{
Congenital Lobar Emphysemma: A Rare Case Report
}

\author{
Siba Shankar Beriha, Pradeep S, AbinashiSabyasachiSethy, Leena Das
}

\begin{abstract}
We describe case of congenital lobar emphysema presented as a case of pneumoniawith pneumothorax in a newborn. Later on with HRCT we found to have left upperlobe emphysema for which the left upper lobectomy was done. After surgery the patient improved dramcatically, successfully extubated and discharged.
\end{abstract}

Key words: congenital lobar emphysema, lobectomy, neonates

\section{Introduction}

Congenital lobar emphysema (CLE) is a rare malformation of the lung that causes respiratory distress in newborn. Wedescribe a case of CLEpresented as a case pneumonia with pneumothroax and found to have CLE which was successfully resected.

\section{Case description}

A full term, $1^{\text {st }}$ order, normally born male child without any significant perinatal problems presented to us on $27^{\text {th }}$ day of life with history of cough and cold for 4 days followed by respiratory distress $\&$ poor feeding for 2 days, without any other significant history.On examination baby was tachypnoeic without tachycardia, withsub costal and intercostals retractions. On auscultation, there were decreased breath sounds on left hemithroax with bilateral crepitations. Saturation was $88 \%$ with 5 litres of Oxygen, cardiovascularsystem revealing well palpable pulse and the shift of apex impulse to right, others were within normal limits

With this clinical picture, bronchopneumonia was suspected and treated with antibiotics. Even with antibiotics the respiratory distress was not subsided. Chest radiograph of the child revealed hyper-lucent left hemithorax with shift of mediasteanum (Figure 1). Other investigations were normal. HRCT (Figure 2) was done found to have emphysematous changes in the left upper lobe and right middle lobe with retrosternal herniation of the left lung to right, with mediastinal shift to right- Congenital lobar emphysema. Patient was successfully operated for lobar emphysema by left thoracotomy and left upper lobectomy (Figure 3). Tissue biopsy sent for histo-pathological examination revealing emphysema. The child was ventilated for $12 \mathrm{hrs}$ and dramatically improved after surgery (Figure 4), then successfully discharged.

\section{Discussion}

Congenital lobar emphysema (CLE) is a rare malformation of the lung that may cause persistent respiratory distress in infants ${ }^{1}$. Prevalence of CLE is 1 in 20,000 to 1 in 30,000 live births. ${ }^{2,3}$ Most of cases presents during neonatal period; but can present up to 6months of age (At birth: $33 \%$, by 1 month: $50 \%$, after 6 month: 5\%). More common in male children (M: $\mathrm{F}=3: 1)$ Upper lobes are predominantly involved ${ }^{4}$. Around 14$21 \%$ is associated other congenital malformations like Congenital heart disease (CHD), rib cage defects and renal defects. In our case, CLE was on left upper lobe, without any other congenital malformations.

Aetiology of CLE is 50\% idiopathic and other proposed etiologies like bronchial cartilage defect (25\%), endobronchial obstruction (13\%), extrinsic bronchial compression by a vascular structure and intrinsic alveolar disease. Pathogenesis of CLEis mostly due to deficiency of bronchial cartilage and airway collapse creating a 'ball-valve' mechanism and air trapping only during expiration.Over distension of the pulmonary lobe is secondary to partial bronchial obstruction ${ }^{1}$. There is gross over inflation of the affected lobe, compression of the remainder of the ipsilateral lung, mediastinal shift, and herniation of the emphysematous lobe into the opposite side of the chest.

CLE mostly presents as progressive respiratory distress with or without cyanosis. CLE usually associated with respiratory tract infections and failure to thrive. Respiratory distress precipitated by feeding, crying, or excitement ${ }^{5}$. On examination, there is chest wall retractions, hyper-resonance on percussion, diminished breath sounds on auscultation, mediastinal shifting, liver or spleen may be palpable due to flattening of the affected side diaphragm. Our case also presented with respiratory distress as bronchopneumonia, but later on found to have reduced breath sounds with resonance note on percussion.

Investigations are mainly radiological, chest radiograph showing large, space-occupying air-filled lobe, collapse of ipsilateral lung, mediastinal shift, and atelectasis of contra lateral lung. High resolution CT (HRCT) scan to exclude other causes and may identify the obstruction, if intraluminal or extrinsic. ECHO in case of associated CHD. CLE can be diagnosis prenatally with fetal sonographyshows echogenic lung. After X-ray and HRCT we found to have CLE, then surgery was done. 
Main stay of treatment is surgery (lobectomy) ${ }^{6}$. Early resection allows compensatory lung growth. When multiple lobes are involved, staged lobectomies with removal of the more hyper inflated lobe first have been successful. Early age, associated CHD or severe respiratory symptoms are not contraindications for operation. Without surgery, the mortality rate is $50 \%$. The mortality rate among surgically treated patients is less than $5 \%$

\section{Conclusion}

In spite of the development of advanced diagnostic techniques, CLE is still a diagnostic dilemma for a pediatrician. So, a high index of suspicion is needed to diagnose CLE in neonates who present with a progressive respiratory distress. A differential diagnosis of CLE should always be considered before inserting an ICD in a suspected case of pneumothorax, especially in a neonate.

\section{References:}

[1]. Abel RM, Bush A. Congenital Lung Disease. In: Chernick Victor., editor. Kendig's disorders of the respiratory tract in children. 7th ed. Philadelphia: Saudners; 2006. pp. 280-316.

[2]. Kravitz RM. Congenital malformations of the lung. PediatrClin North Am.1994;41:453-72. [PubMed]

[3]. Parray T, Apuya JS, Abraham E, Ahsan F, Shah S. Anesthesiologist's dilemma in a patient with congenital lobar emphysema. Internet J Anesthesiol. 2010;24:1

[4]. Floyd FW, Repici AJ, Gibson ET, Mcgeorge CK. Bilateral Congenital labor emphysema surgically corrected. Pediatrics. 1963;1:87-96. [PubMed]

[5]. Bush A. Prenatal presentation and postnatal management of congenital thoracic malformations. Early Hum Dev. 2009;85:67984. [PubMed]

[6]. Choudhury SR, Chadha R, Mishra A, Kumar V, Singh V, Dubey NK. Lung resections in children for congenital and acquired lesions. PediatrSurg Int.2007;23:851-9. [ [PubMed]

Figure 1

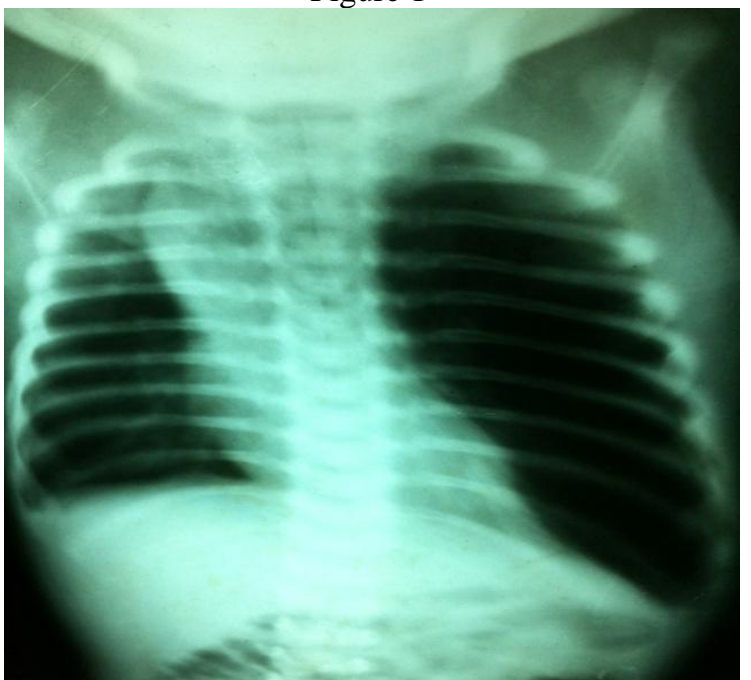

Chest radiograph of the child showing the mediastinal shifting and hyper inflat

Figure 2

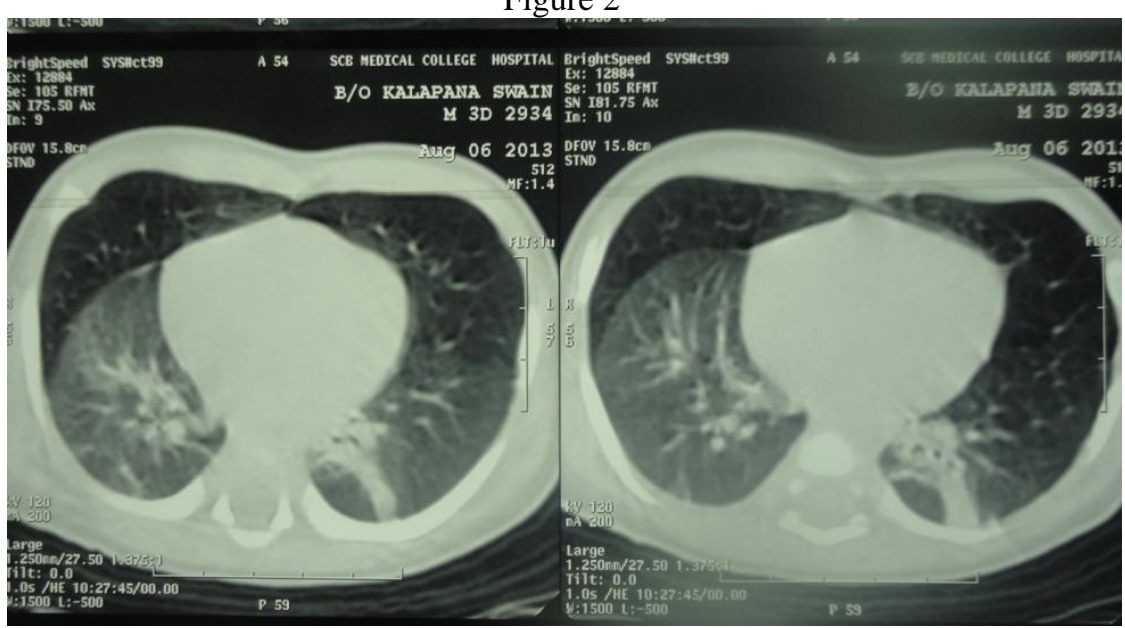


Left lung showing emphysematous changes and retrosternal herniationof left lung into right side of thorax. Figure 3

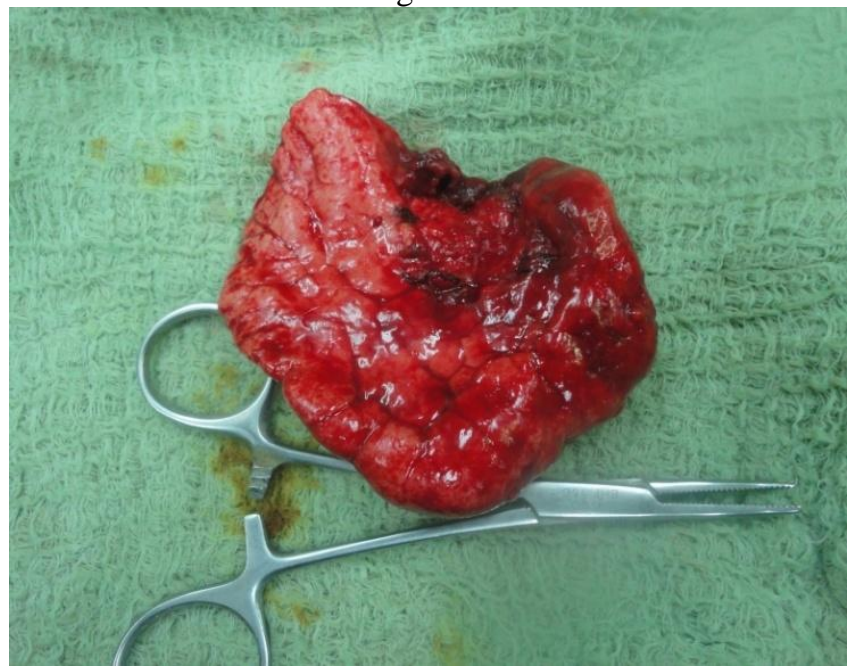

Resected specimen showing upper lobe of left lung

Figure 4

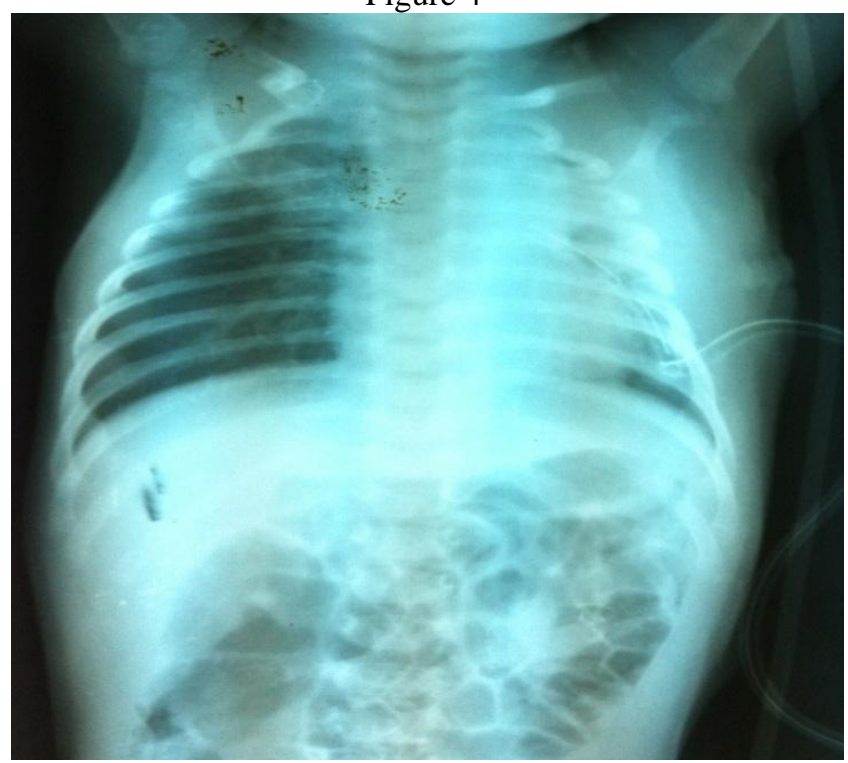

Chest radiograph showing mediastinum in midline and chest tube in place. 